\title{
Frequency and significance of indeterminate and borderline Quantiferon Gold TB IGRA results
}

\author{
To the Editor:
}

The Quantiferon Gold test is one of two commercially available tuberculosis (TB) interferon- $\gamma$ release assays (IGRAs) recommended for the diagnosis of latent TB infection [1]. A Quantiferon Gold test is considered positive if the result is $\geqslant 0.35 \mathrm{IU} \cdot \mathrm{mL}^{-1}$ [2]. However, longitudinal studies have shown that a significant number of test reversions and conversions occur if results just above or below this threshold are repeated $[3,4]$. This variation may be due to random chance or other factors such as test-related errors, differences in absolute lymphocyte numbers and within-subject variability of the interferon- $\gamma$ response $[5,6]$. The high proportion of conversions and reversions around the $0.35 \mathrm{IU} \cdot \mathrm{mL}^{-1}$ cut-off has led some to suggest that a borderline or equivocal range should be used [7], as this avoids treatment of individuals who do not have a 'stable' positive result, as well as failure to treat those just below the 0.35 threshold who would convert to a positive result if the test were repeated [6].

Two versions of the Quantiferon Gold test are currently available: the Gold In-Tube and Gold Plus. The latter was introduced in 2016. Despite some technical differences, they use similar test platforms and give equivalent readouts $[8,9]$.

Various cut-offs have been proposed for a Quantiferon equivocal range: European studies and a systematic review have suggested $0.2-0.7 \mathrm{IU} \cdot \mathrm{mL}^{-1}[10-13]$, as conversions have been shown to occur most frequently when the first IGRA result is between $0.2-0.35 \mathrm{IU} \cdot \mathrm{mL}^{-1}$, and reversions when the initial result is between $0.35-0.7 \mathrm{IU} \cdot \mathrm{mL}^{-1}$ [14]. On the other hand, American workers have suggested ranges including $0.1-1.0 \mathrm{IU} \cdot \mathrm{mL}^{-1}$ [7], $0.35-1.1 \mathrm{IU} \cdot \mathrm{mL}^{-1}$ and $0.35-2.0 \mathrm{IU} \cdot \mathrm{mL}^{-1}[15,16]$. In our centre, we used the 0.2-0.7 IU $\mathrm{mL}^{-1}$ range in line with other low-incidence TB European settings.

In addition to borderline results, Quantiferon tests may be uninterpretable if either the positive (mitogen) or negative control tubes fail to give an appropriate response. Such results are termed 'indeterminate' and the test may need to be repeated.

To evaluate the proportion of Quantiferon tests performed in clinical practice, which do not give a clear positive or negative result, and the yield of repeating indeterminate or equivocal tests, we undertook a retrospective review of all individuals undergoing Quantiferon Gold testing between 2010 and 2014 at the Royal Free London NHS Foundation Trust, London, UK. This retrospective study specifically evaluated indeterminate and equivocal results, and the yield of repeating these; tests that gave a clearly positive or negative result were not repeated.

In all, we identified 3002 subjects who underwent Quantiferon Gold testing. Of these, 1752 (58.4\%) were female, and the median (interquartile range) age was $32(25-44)$ years. Indications for testing were: routine occupational health screening in 1328 individuals (44.2\%); work-up prior to biological therapy in 1185 (39.5\%) and screening of contacts of active TB cases in 291 (9.7\%). The latter group was tested within 6 months of contact with active TB. Three individuals had Quantiferon tests as part of new entrant (migrant) screening, and 195 (6.5\%) were tested for other reasons, or the indication for testing was not recorded.

Using a $0.35 \mathrm{IU} \cdot \mathrm{mL}^{-1}$ cut-off, 390 of 3002 results (13\%) were positive, 2485 (83\%) negative and $84(3 \%)$ were indeterminate. If an equivocal range of $0.2-0.7$ was used, $2412(80 \%)$ results were negative $(<0.2), 342$

@ERSpublications

Borderline and equivocal results for the Quantiferon Gold test are common and repeated borderline tests often change http://ow.ly/PDDZ30fqJ1c

Cite this article as: Brown J, Kumar K, Reading J, et al. Frequency and significance of indeterminate and borderline Quantiferon Gold TB IGRA results. Eur Respir J 2017; 50: 1701267 [https://doi.org/10.1183/ 13993003.01267-2017]. 
(11\%) were positive $(>0.7)$, and $156(5 \%)$ had values lying in the equivocal range (figure 1$)$. Of the 130 equivocal tests for which numerical results were available, $56(43 \%)$ were $\geqslant 0.35 \mathrm{IU} \cdot \mathrm{mL}^{-1}$ and $73(56 \%)$ were $<0.35 \mathrm{IU} \cdot \mathrm{mL}^{-1}$ (27 results were in the $0.2-0.7$ range and reported as equivocal, but the absolute value was not available).

84 tests (3\%) were indeterminate, primarily due to a lack of response in the mitogen tube (71 of 73 with numerical results available). Individuals tested prior to initiation of biological (e.g. anti-TNF) therapy were more likely to have an indeterminate result than other groups: $6 \%(66 / 1185)$ had an indeterminate result, compared to $0.5 \%(6 / 1328)$ of healthcare workers that were tested (Chi-squared test, $\mathrm{p}<0.001)$.

107 individuals with an initial equivocal or indeterminate result had a repeat test undertaken within 6 months of the original test. Of the 107 repeat tests, 56 (52\%) were negative, 14 (13\%) positive, 25 (23\%) equivocal, $10(9 \%)$ indeterminate, and two could not be analysed owing to laboratory processing issues such as the tubes being under-filled or the wrong tubes being sent for analysis (figure 1).

To evaluate the usefulness of an equivocal range for borderline results, we specifically looked at the 69 tests with initial results in the equivocal range that were repeated within 6 months: 34 (49\%) of these repeat tests were negative, $24(35 \%)$ were again equivocal and $11(16 \%)$ were positive. If an equivocal range was not used and a cut-off of 0.35 applied to these repeat results, 49 (71\%) repeat tests were negative and 20 (29\%), positive.

Of the 38 initial tests with results in the equivocal range below the 0.35 cut-off (i.e. $0.2-0.34)$ which were repeated, seven $(18 \%)$ had a positive repeat test $(\geqslant 0.35)$ of which five $(13 \%)$ were above the higher 0.7 threshold. In contrast, of the 28 individuals with an initial equivocal result over $0.35,16$ (57\%) were negative $(<0.35)$ on repeat testing ( 11 had results below 0.2 , and five had results between 0.2 and 0.34$)$ and $12(43 \%)$ were positive $(>0.35)$ on repeat testing (five had results above 0.7 , and seven had results between 0.35 and 0.7 ). In three individuals documented as having an initial equivocal result which was then repeated, a numerical value for the equivocal result was not available. 35\% (23/66) of results in the equivocal range therefore changed when repeated.

In conclusion, although the Quantiferon Gold test seeks to provide a positive or negative result, in routine clinical practice, $8 \%$ of tests are either not interpretable or lie in an "equivocal" range where the risk of progression to active TB is likely different to those results which are clearly positive or negative [17]. Our data therefore lend support to the use of an equivocal range for the reporting of Quantiferon tests: nearly one-fifth of results just below the 0.35 cut-off were positive when repeated, and a half of those just above the 0.35 threshold were negative when repeated. This suggests that relying on a single result within this equivocal range may result in both over-treatment and under-treatment of patients.

This study has several limitations. In particular, we relied on the retrospective use of routinely collected clinical data, and not all equivocal or indeterminate results were repeated. Individuals with a first test that was positive or negative did not have repeat tests, so we are unable to evaluate the stability of these results.

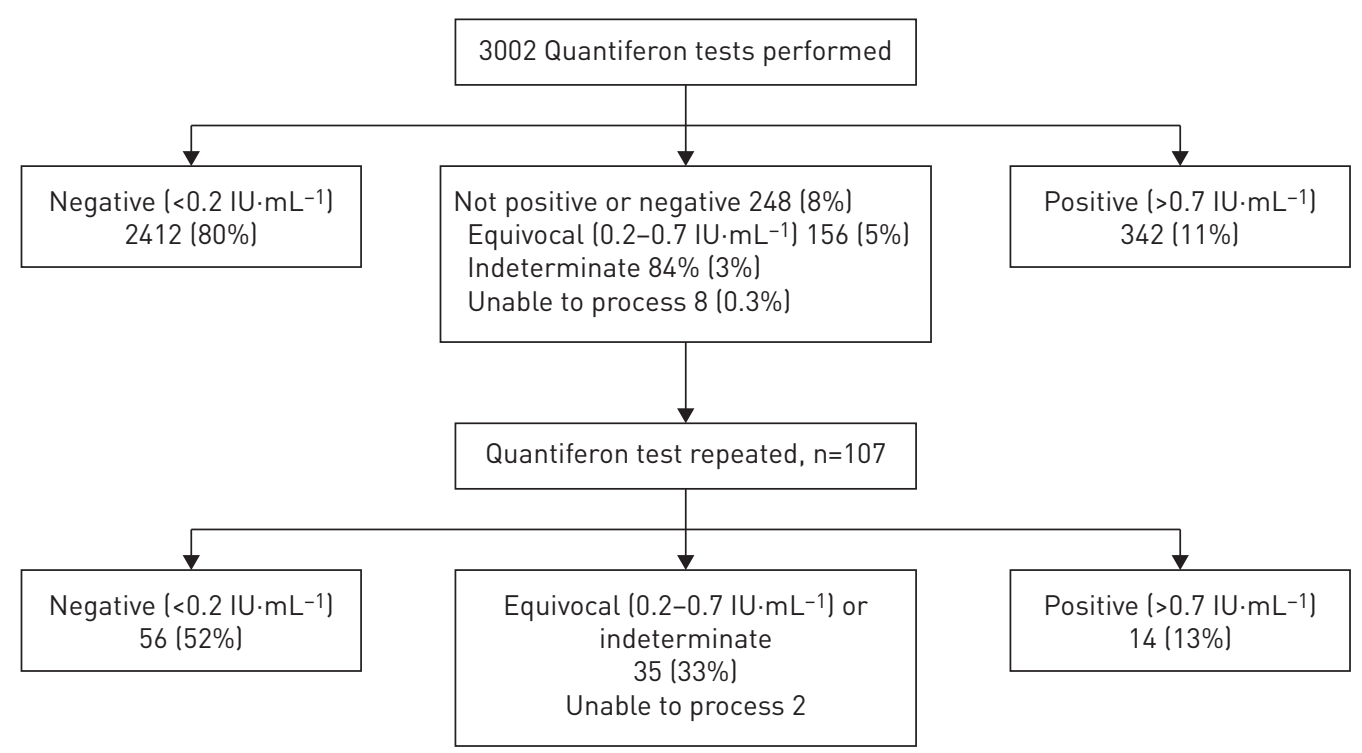

FIGURE 1 Flow chart of results. 
Where test values reverted from positive to negative around the $0.35 \mathrm{IU} \cdot \mathrm{mL}^{-1}$ threshold, tests were not repeated a third time; therefore, we cannot determine if these represent 'stable' reversions.

The relatively high frequency of indeterminate results in our cohort is likely to be a consequence of the high proportion of individuals (39\%) tested prior to receiving biological therapies (e.g. anti-TNF) and may therefore have been using immunosuppressant treatments or had conditions that could affect their immune responses, as there were a significantly higher proportion of indeterminate results in this group than in the healthcare workers tested. This reinforces the need to specifically evaluate the performance of such tests in immunocompromised groups who may be at greater risk of progression to active TB, particularly given that Quantiferon tests would be of most value in this subpopulation.

The recently introduced Quantiferon Gold Plus test contains an additional patient tube seeking to elicit CD8 in addition to CD4 lymphocyte responses $[8,9]$. This modification might in fact increase the risk of borderline test results, given that there are now two TB antigen tubes rather than one. As newer immunodiagnostic tests for tuberculosis infection enter clinical practice, evaluation of the frequency of indeterminate and borderline results will remain important, particularly as the cut-offs used may affect the number of people offered treatment for latent TB infection, which in turn will impact on the cost and yield of such programmes.

In summary, indeterminate or equivocal results from the Quantiferon Gold test occur in $8 \%$ of samples tested, and results in the borderline range changed from positive to negative or vice versa in $35 \%$ of cases when repeated. This supports the use of an equivocal range for the Quantiferon assay.

James Brown ${ }^{1,2,5}$, Kartik Kumar ${ }^{3,5}$, Jacob Reading ${ }^{4}$, Jennifer Harvey ${ }^{1}$, Saraswathi Murthy ${ }^{1}$, Santino Capocci ${ }^{1}$, Susan Hopkins ${ }^{1}$, Suranjith Seneviratne ${ }^{1}$, Ian Cropley ${ }^{1}$ and Marc Lipman ${ }^{1,2}$

${ }^{1}$ Royal Free London NHS Foundation Trust, London, UK. ${ }^{2}$ UCL Respiratory, Division of Medicine, University College London, London, UK. ${ }^{3}$ North Middlesex University Hospital NHS Trust, London, UK. ${ }^{4}$ John Radcliffe Hospital, Oxford University Hospitals NHS Foundation Trust, Headington, Oxford, UK. ${ }^{5}$ Joint first authors.

Correspondence: James Brown, Royal Free London NHS Foundation Trust, Pond Street, Hampstead, London, NW3 2QG, UK. E-mail: james.brown13@nhs.net

Received: June 262017 | Accepted after revision: Aug 072017

Conflict of interest: None declared.

Acknowledgements: We would like to thank Ronnie Chee, Mark Davis and Annabelle Mai for assistance with the recording, distribution and supervision of results.

\section{References}

1 National Institute for Health and Care Excellence. Tuberculosis. NICE guidelines. London, NICE, 2016

2 Connell TG, Rangaka MX, Curtis N, et al. QuantiFERON-TB Gold: state of the art for the diagnosis of tuberculosis infection? Expert Rev Mol Diagn 2006; 6: 663-677.

3 Schablon A, Nienhaus A, Ringshausen FC, et al. Occupational screening for tuberculosis and the use of a borderline zone for interpretation of the IGRA in German healthcare workers. PLoS One 2014; 9: e115322.

4 Pai M, O'Brien R. Serial testing for tuberculosis: can we make sense of $\mathrm{T}$ cell assay conversions and reversions? PLoS Med 2007; 4: e208.

5 Detjen AK, Loebenberg L, Grewal HM, et al. Short-term reproducibility of a commercial interferon gamma release assay. Clin Vaccine Immunol 2009; 16: 1170-1175.

6 van Zyl-Smit RN, Zwerling A, Dheda $\mathrm{K}$, et al. Within-subject variability of interferon- $\gamma$ assay results for tuberculosis and boosting effect of tuberculin skin testing: a systematic review. PLoS One 2009; 4: e8517.

7 Fong KS, Tomford JW, Teixeira L, et al. Challenges of interferon-gamma release assay conversions in serial testing of health-care workers in a TB control program. Chest 2012; 142: 55-62.

8 Barcellini L, Borroni E, Brown J, et al. First evaluation of QuantiFERON-TB Gold Plus performance in contact screening. Eur Respir J 2016; 48: 1411-1419.

9 Petruccioli E, Chiacchio T, Pepponi I, et al. First characterization of the CD4 and CD8 T-cell responses to QuantiFERON-TB Plus. J Infect 2016; 73: 588-597.

10 Torres Costa J, Silva R, Sa R, et al. Serial testing with the interferon-gamma release assay in Portuguese healthcare workers. Int Arch Occup Environ Health 2011; 84: 461-469.

11 Schablon A, Harling M, Diel R, et al. Serial testing with an interferon-gamma release assay in German healthcare workers. GMS Krankenhhyg Interdiszip 2010; 5: Doc05.

12 Schablon A, Diel R, Diner G, et al. Specificity of a whole blood IGRA in German nursing students. BMC Infect Dis 2011; 11: 245 .

13 Ringshausen FC, Schablon A, Nienhaus A. Interferon-gamma release assays for the tuberculosis serial testing of health care workers: a systematic review. J Occup Med Toxicol 2012; 7: 6.

14 Nienhaus A, Ringshausen FC, Costa JT, et al. IFN-gamma release assay versus tuberculin skin test for monitoring TB infection in healthcare workers. Expert Rev Anti Infect Ther 2013; 11: 37-48.

15 Thanassi W, Noda A, Hernandez B, et al. Delineating a retesting zone using receiver operating characteristic analysis on serial QuantiFERON tuberculosis test results in US healthcare workers. Pulm Med 2012; 2012: 291294. 
16 Joshi M, Monson TP, Woods GL. Use of interferon-gamma release assays in a health care worker screening program: experience from a tertiary care centre in the United States. Can Respir J 2012; 19: 84-88.

17 Hermansen TS, Lillebaek T, Langholz Kristensen K, et al. Prognostic value of interferon-gamma release assays, a population-based study from a TB low-incidence country. Thorax 2016; 71: 652-658.

Copyright @ERS 2017 